\title{
Effects of Differential Stretching Protocols DURING WARM-UPS ON High-SPEed MOTOR Capacities in Professional Soccer Players
}

\author{
Thomas Little ${ }^{1}$ and Alun G. Williams ${ }^{2}$ \\ ${ }^{1}$ Sport, Health, and Exercise, Staffordshire University, Staffordshire, UK; ${ }^{2}$ Institute for Biophysical and Clinical \\ Research into Human Movement, Manchester Metropolitan University, Alsager, UK.
}

\begin{abstract}
Little, T., and A.G. Williams. Effects of differential stretching protocols during warm-ups on high-speed motor capacities in professional soccer players. J. Strength Cond. Res. 20(1):203-207. 2006. - The purpose of this study was to examine the effects of different modes of stretching within a pre-exercise warm-up on high-speed motor capacities important to soccer performance. Eighteen professional soccer players were tested for countermovement vertical jump, stationary $10-\mathrm{m}$ sprint, flying 20-m sprint, and agility performance after different warm-ups consisting of static stretching, dynamic stretching, or no stretching. There was no significant difference among warm-ups for the vertical jump: mean $\pm S D$ data were $40.4 \pm 4.9 \mathrm{~cm}$ (no stretch), $39.4 \pm 4.5 \mathrm{~cm}$ (static), and $40.2 \pm 4.5 \mathrm{~cm}$ (dynamic). The dynamic-stretch protocol produced significantly faster $10-\mathrm{m}$ sprint times than did the no-stretch protocol: $1.83 \pm 0.08$ seconds (no stretch), $1.85 \pm 0.08$ seconds (static), and $1.87 \pm 0.09$ seconds (dynamic). The dynamic- and static-stretch protocols produced significantly faster flying $20-\mathrm{m}$ sprint times than did the nostretch protocol: $2.41 \pm 0.13$ seconds (no stretch), $2.37 \pm 0.12$ seconds (static), and $2.37 \pm 0.13$ seconds (dynamic). The dynamic-stretch protocol produced significantly faster agility performance than did both the no-stretch protocol and the staticstretch protocol: $5.20 \pm 0.16$ seconds (no stretch), $5.22 \pm 0.18$ seconds (static), and $5.14 \pm 0.17$ seconds (dynamic). Static stretching does not appear to be detrimental to high-speed performance when included in a warm-up for professional soccer players. However, dynamic stretching during the warm-up was most effective as preparation for subsequent high-speed performance.
\end{abstract}

KEY WoRDs. power, stretch, football

\section{INTRODUCTION}

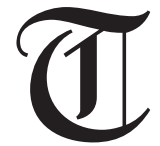

he inclusion of static stretching as part of a preexercise warm-up routine has been commonplace in a multitude of sports, including soccer. This stemmed from the belief that static stretching will aid performance and decrease injury risk (1). However, new research has challenged some longheld concepts about common stretching practices. In particular, research suggests that a regimen of stretching provides an acute inhibition of maximal force production by the stretched muscle. Bouts of static stretching reduced the force capacity during various strength tests ( 7 , $13,15,17,21)$ and impaired vertical jump performance in some cases $(5,23)$ but not in another $(20)$. Changes in mechanical properties of the musculotendon unit or changes in neural activation may decrease force. Nelson et al. (15) suggested that increased muscular compliance as a result of stretching might mean the muscle will go through a greater period of unloaded shortening before taking up slack sufficiently to transfer generated force to the bone. Consequently, cross-bridges may be at a less optimal length much sooner in the full range of motion (ROM).

Findings that stretching is detrimental to force production have prompted recommendations that static stretching be omitted or replaced by dynamic stretching during warm-ups $(2,14,18)$. These recommendations based on existing research evidence may be premature, as the effects of static stretching on performance in a sporting environment and with elite sporting competitors remain unclear. Furthermore, despite use of dynamic stretching by some sports participants, scant research exists on the effects of this mode of stretching on physical performance parameters closely related to the actual demands of sport.

Soccer is a sport requiring high-intensity, intermittent, noncontinuous exercise (8) that includes many sprints of different durations, rapid acceleration, jumping, agility, and so on. However, to date, no research has investigated the effects of different stretching modes on physical performance in soccer players. Thus, we aimed to test the hypotheses that static stretching is detrimental to performance and that dynamic stretching is beneficial to performance compared with static stretching or no stretching. Therefore, the specific purpose of this study was to examine the effects of static and dynamic stretching within a warm-up on acceleration, maximal speed, vertical jump, and agility of professional soccer players.

\section{MeTHODS}

\section{Experimental Approach to the Problem}

In a within-subjects experimental design, professional soccer players conducted 3 different warm-up protocols on 3 nonconsecutive test days within 1 week. Each test day occurred more than 48 hours after a match or hard physical training to minimize the fatiguing effects of previous exercise. The warm-up protocols differed only in the mode of stretching used, whereas all other exercises used in the warm-up were identical. The stretching modes used were static stretch, no stretch, and dynamic stretch. Performance tests of acceleration, maximal running speed, vertical jump, and agility were conducted after each warmup protocol.

\section{Subjects}

Eighteen professional soccer players from an English League Premier Division club were tested during the 2001-02 season as part of their athletic training program. All subjects gave their informed consent, and the Local 
Ethics Committee approved this study. All subjects were familiar with all testing procedures used in this study before the experiment began, as the tests were routinely used in the monitoring of their fitness status.

\section{Procedures}

Three warm-up protocols differentiated by their stretching content were used: static, no stretch, and dynamic. Subjects conducted these 3 protocols on 3 separate days in that order. Aside from the stretching, each warm-up followed the exact same procedure, consisting of the following:

- 4 minutes of jogging and varied movements, including 2 minutes of jogging, 1 minute of sidestepping and back jogging, and 1 minute of further jogging.

- 6.20 minutes of flexibility exercises (except for nostretch protocol).

- $\sim 4$ minutes of incremental intermittent sprint and agility runs. These initially included three-quarter pace running: $10 \mathrm{~m}$ forward and $5 \mathrm{~m}$ sidestepping, repeated twice; $30 \mathrm{~m}$ forward, repeated 3 times; and $45 \mathrm{~m}$ forward with $5 \times 90^{\circ}$ changes of direction, repeated twice. Intensity was then increased: three-quarter pace for 10 $\mathrm{m}$ and full pace for $20 \mathrm{~m}$, repeated twice, and full pace for $30 \mathrm{~m}$.

- 2 minutes of rest.

The principal locomotive leg muscle groups were stretched (gastrocnemius, hamstrings, quadriceps and hip flexors, gluteals, adductors). The static stretches used were \#21 (gastrocnemius), \#69 (hamstrings, modified with subject holding own leg), \#101 (hip flexor and quadriceps, modified with vertical thigh and trunk alignment) and \# 114 (gluteals) described by Alter (1), and the saddle (adductors) described by Hoffman (12). A 20 -second rest was allowed between each stretch. For static stretching, subjects held the stretch for 30 seconds on each leg before changing immediately to the contralateral side. Subjects were told to stretch until they approached the end of the ROM but within the pain threshold. Subjects performed the dynamic stretches on alternate legs for 60 seconds at a rate of approximately 1 stretch cycle every 2 seconds or unilaterally for 30 seconds, then they repeated on the other leg at a rate of approximately 1 stretch cycle every second. The dynamic stretches used were the backwardreach run (quadriceps); lateral lunge (adductors); drop lunge (gluteals) and straight-leg march (hamstrings) described in Fredrick and Szymanski (11); and the heel-totoe walk (gastrocnemius), where the subject landed in maximal dorsiflexion and moved to maximal plantar flexion with each step. Subjects were instructed to try and attain maximal ROM with each repetition. In the nostretch protocol, instead of stretching, subjects rested for 1 minute after the general warm-up and then proceeded to complete the incremental intermittent sprint and agility runs.

All performance tests were performed on an indoor synthetic pitch. Vertical jump, stationary 10 -m sprint, flying $20-\mathrm{m}$ sprint, and a zig-zag course were used to assess leg power, acceleration, maximal speed, and agility capacities. Newstart Electronic timing gates and jumping mat (Brower Timing System, Salt Lake City, UT) were used to measure run completion times and vertical jump, respectively. Vertical jump involved a 2 -footed countermovement jump from a stationary position with the in-

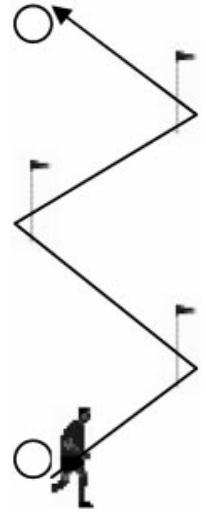

FigURE 1. Diagram of the course used in the agility test. Each straight sprint is $5 \mathrm{~m}$ and each turn at a flag is $100^{\circ}$.

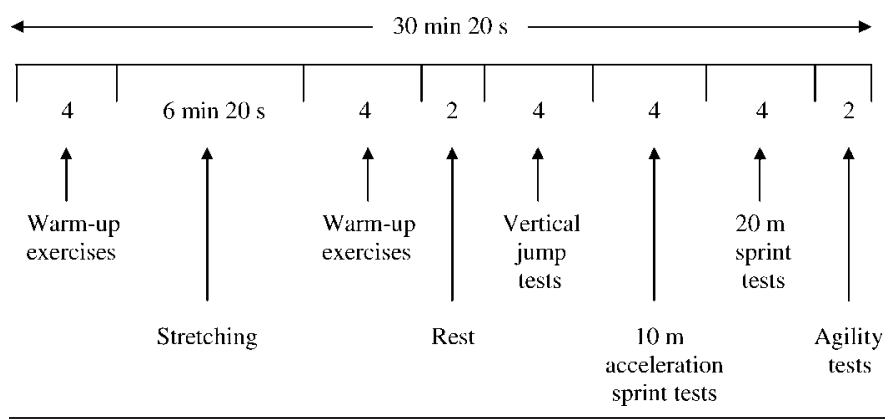

Figure 2. Timeline during each data-collection session.

tention of attaining maximum height. Subjects were instructed to maintain hands on the hips and keep the legs straight once they had left the ground. Test-retest ratio limits of agreement (16) data were 1.021./1.096 (bias not significant), showing good reliability of the vertical jump test. Stationary $10-\mathrm{m}$ sprint involved sprinting $10 \mathrm{~m}$ as fast as possible from a stationary start position. Testretest ratio limits of agreement were 0.999./1.042 (bias not significant), showing very good reliability of the $10-\mathrm{m}$ acceleration sprint. Flying $20-\mathrm{m}$ sprint involved sprinting $20 \mathrm{~m}$ as fast as possible from a maximal-speed start. Testretest ratio limits of agreement were 0.997./1.040 (bias not significant), showing very good reliability of the $20-\mathrm{m}$ maximal sprint. The $20-\mathrm{m}$ zig-zag course included three $100^{\circ}$ turns at 5 -m intervals (Figure 1 ). This test was chosen because of its relative simplicity and the fact that the subjects were all very familiar with the test (having conducted it approximately every 6 weeks during the current and previous seasons), which meant that the learning effects would be minimal. Test-retest ratio limits of agreement were 1.003./1.022 (bias not significant), showing very good reliability of the agility test. During the test session, each subject first performed the vertical jump test, followed by the stationary $10-\mathrm{m}$ sprint test, the flying $20-\mathrm{m}$ sprint test, and finally the agility test. Subjects performed 2 maximal attempts at each exercise, and the best time was retained for analysis. A rest of 2 minutes between trials and tests was included to minimize the effects of fatigue. A timeline for the procedure on a test day is shown in Figure 2. 


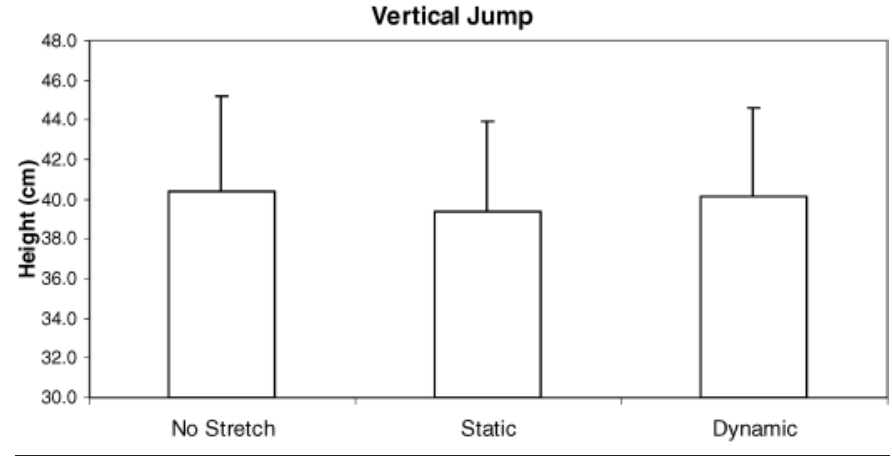

FIGURE 3. Vertical jump measures for the different warm-up protocols.

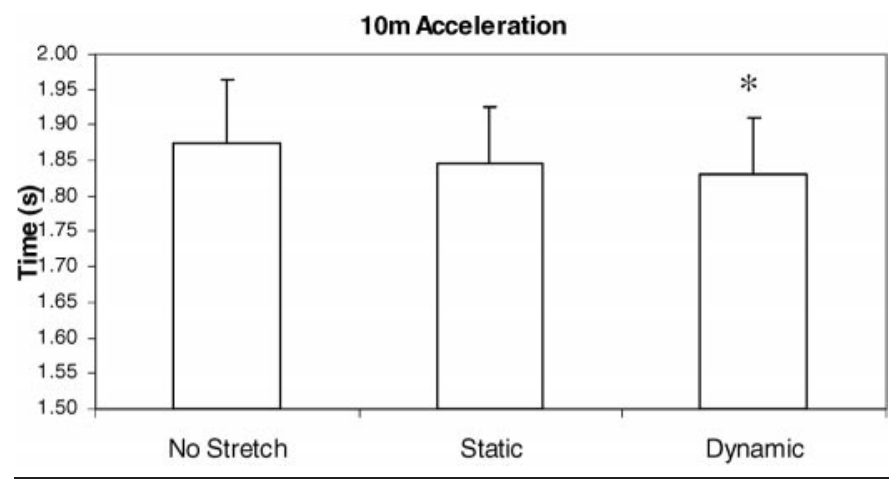

Figure 4. Ten-meter acceleration sprint times for the different warm-up protocols. * Significantly faster than the nostretch condition $(p=0.011)$.

\section{Statistical Analyses}

Repeated-measures analysis of variance (ANOVA) was used to compare the performances after the 3 different warm-ups. Newman-Keuls post hoc analysis was used to identify pairwise differences. Statistical significance was accepted at $p \leq 0.05$.

\section{Results}

The mean scores $( \pm S D)$ for the performance measures after the different warm-up procedures are presented in Figures 3-6.

There was no significant difference among the different warm-up protocols for vertical jump performance, as shown in Figure 3 (ANOVA $p=0.074$ ). There were significant differences among the different warm-up protocols for acceleration (Figure 4), with dynamic stretching resulting in significantly superior performance than no stretching (ANOVA $p=0.025$, no stretch vs. static $p=$ 0.079 , no stretch vs. dynamic $p=0.011$, static vs. dynamic $p=0.350$ ). In tests for maximal speed (Figure 5), static and dynamic stretching produced significantly better performance than did no stretching (ANOVA $p<0.0005$, no stretch vs. static $p<0.0005$, no stretch vs. dynamic $p<$ 0.0005 , static vs. dynamic $p=0.921$ ). There were significant differences among the warm-up protocols for agility (Figure 6), with dynamic stretching resulting in significantly better performance than static stretching and no stretching (ANOVA $p<0.0005$, no stretch vs. static $p=$ 0.232 , no stretch vs. dynamic $p=0.001$, static vs. dynamic $p<0.0005$ ).

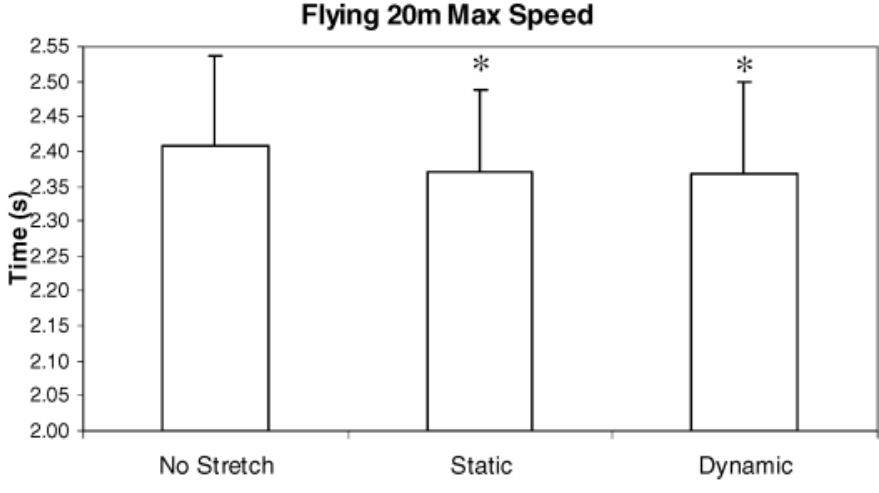

Figure 5. Twenty-meter maximal-speed times for the different warm-up protocols. * Significantly faster than the nostretch condition (no stretch vs. static $p<0.0005$, no stretch vs. dynamic $p<0.0005$ ).

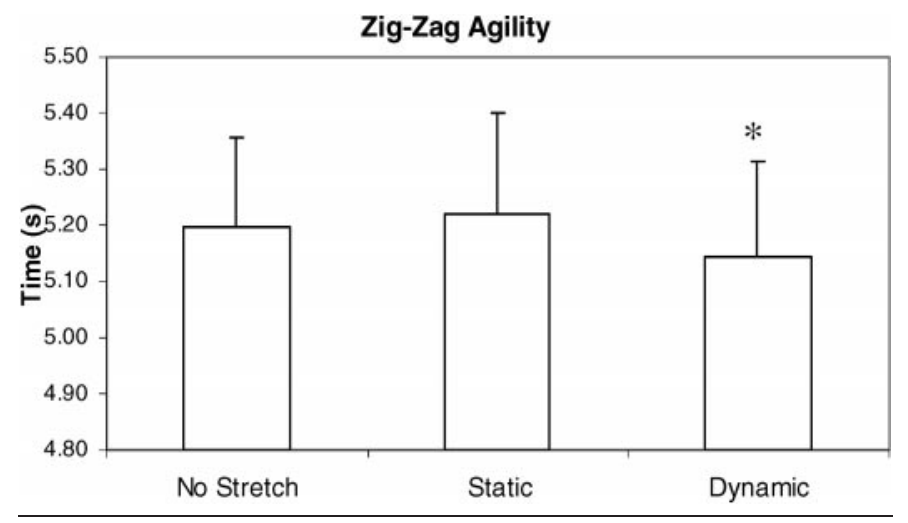

FigURE 6. Zig-zag agility times for the different warm-up protocols. * Significantly faster than the no-stretch and static conditions (no stretch vs. dynamic $p=0.001$, static vs. dynamic $p<0.0005$ ).

\section{Discussion}

For 3 of the 4 measures used, there was no difference between static- and no-stretch warm-up protocols, whereas in the $20-\mathrm{m}$ maximal-speed test the static-stretch protocol produced significantly faster runs than did the nostretch protocol. Thus, our data do not support the hypothesis that static stretching has a detrimental effect on high-speed performance in professional soccer players under the experimental conditions used in this study. Dynamic stretching also produced better performance than did no stretching in 3 of the 4 tests used. Consequently, our data support the hypothesis that dynamic stretching is beneficial compared with no stretching. Dynamic stretching produced better performance than did static stretching in 1 of the 4 tests used (agility), and the performance data on 2 of the other 3 tests also showed a tendency for a beneficial effect of dynamic stretching (maximal 20-m sprint performance was identical after the static- and dynamic-stretch protocols). Therefore, we also believe that our data tentatively support the hypothesis that dynamic stretching is beneficial when compared with static stretching for professional soccer players under the experimental conditions used in this study.

Dynamic stretching resulted in the best scores in all but the vertical jump test; therefore, used within precompetition warm-ups, it is probably optimal for the high- 
speed performances required in sports such as soccer. Dynamic stretching is an active contractile process, and the performance benefits obtained may stem from facilitated motor control (6) via rehearsal of specific movements, increased muscle blood flow, or elevated core or peripheral temperature, which may increase the sensitivity of nerve receptors and increase the speed of nerve impulses, potentially encouraging muscle contractions to be more rapid and forceful (19). Thus, the improvements in performance may have resulted from either the effects of stretching per se, improved neural function, or additional metabolic cost.

Discrepancies between the current study design and that of previous studies may explain why the static stretching produced no performance decrements in the present study. Previous protocols $(4,10,13,15,17)$ have usually stretched muscles for greater durations than those used in common precompetition warm-ups. Such stretching durations may elicit neural and excessive mechanical force inhibitory mechanisms $(10,15,21)$ that are not apparent during common precompetition warm-ups. By using 30-second stretch durations, we may have avoided this effect. Also, performance analysis has often been conducted immediately after stretching (4, 5, 9, 13, 15, 17). In practice in sport, there is often further warm-up activity after the stretching (as in the current study). Rosenbaum and Hennig (18) reported that additional physical activity after stretching attenuated the decreases in peak force, rate of force development (RFD), relaxation rate, and electromyography amplitudes of the Achilles tendon reflex. Extra muscle activity after stretching may have reversed any decrease in muscular compliance and associated decreased neural drive initiated by stretching. However, it is not yet clear whether these improvements in performance were attributed to the effects of the extra activity itself or the increased time delay from stretching until the measured performance. Furthermore, research indicating that static stretching is detrimental to performance has often involved relatively slow actions such as 1 repetition maximum tests and isokinetic testing $(13,15$, 17 ), yet most important motor actions in soccer, such as sprinting and shooting, are dependent on high-velocity movements and RFD. Findings from Nelson et al. (15) suggest that the deleterious impact of stretching on force production might be limited to movements performed at relatively slow velocities. The authors reported that attenuation in poststretch force decreased as movement speed increased, to the point where at the fastest movement speed (4.71 $\left.\mathrm{rad} \cdot \mathrm{s}^{-1}\right)$ poststretch force was greater, though not significantly. However, vertical jump performance is reliant on high RFD; has been shown to be impaired by static stretching in some studies $(5,21,23)$ but not in another (20); and was worse, though not significantly, after static stretching in the present study. A complicating factor in the present study is that the vertical jump test was the first to be conducted after the stretching protocol (refer to the timeline in Figure 2); therefore, if any detrimental effect of static stretching degrades over time, the vertical jump is most likely to show a detrimental effect in our experiment. The differing effects of static stretching on vertical jump and horizontal speed performance observed in the current study warrant further research.

The use of short-duration static stretching in a soccer precompetition warm-up routine does not appear detri- mental to subsequent high-speed performance. However, the benefit of using static stretching in a warm-up remains questionable. Although research by Pope et al. (17) suggests general static stretching has minimal effect on injury prevention, evidence suggests that static stretching has a greater effect on ROM than does dynamic stretching (3) and that poor ROM is associated with increased risk of injury in soccer players (22). Therefore, static stretching on specific areas of muscular tightness during warm-ups may improve ROM without compromising high-speed performance. However, until research examines further aspects of performance and injury after differential stretching on specific areas of muscular tightness or imbalance, static stretching should not be recommended for warm-ups.

\section{Practical Applications}

Dynamic stretching during warm-ups, as opposed to static stretching or no stretching, is probably most effective as preparation for the high-speed performances required in sports such as soccer. If static stretching is used, evidence suggests that limiting the stretches to short durations and following the stretching with further activity will minimize decrements to power-based performance.

\section{REFERENCES}

1. Alter, M. Science of Flexibility. Champaign, IL: Human Kinetics, 1996.

2. ANDERson, O. Does stretching help prevent injuries? Sports Inj. Bull. 28:6-9. 2003.

3. BANDY, W.D., J.M. IRION, AND M. BRIGGLER. The effect of static stretch and dynamic range of motion training on the flexibility of the hamstring muscles. J. Orthop. Sports Phys. Ther. 27:295300. 1998.

4. Behm, D.G., D.C. Button, AND J.C. Butt. Factors affecting force loss with prolonged stretching. Can. J. Appl. Physiol. 26: 261-272. 2001.

5. Church, J.B., M.S. Wiggins, F.M. Moode, ANd R. CRist. Effect of warm-up and flexibility treatments on vertical jump performance. J. Strength Cond. Res. 15:332-336. 2001.

6. ClaRK, M.A. Integrated Training for the New Millennium. Thousand Oakes, CA: National Academy of Sports Medicine, 2000.

7. Cramer, J.T., T.J. Housh, G.O. Johnson, J.M. Miller, J.W. CoBurn, AND T.W. BECK. Acute effects of static stretching on peak torque in women. J. Strength Cond. Res. 18:236-241. 2004.

8. EkBlom, B. Applied physiology of soccer. Sports Med. 3:50-60. 1986.

9. Fletcher, I.M., AND B. Jones. The effect of different warmup stretch protocols on 20 meter sprint performance in trained rugby union players. J. Strength Cond. Res. 18:885-888. 2004.

10. Fowles, J.R., D.G. Sale, AND J.D. MacDougall. Reduced strength after passive stretch of the human plantarflexors. $J$. Appl. Physiol. 89:1179-1188. 2000.

11. Fredrick, G.A., AND D.J. Szymanski. Baseball (part 1): Dynamic flexibility. Strength Cond. J. 23:21-30. 2001.

12. Hoffman, J. Physiological Aspects of Sports Training and Performance. Champaign, IL: Human Kinetics, 2002.

13. Kokkonen, J., A.G. Nelson, And A. CoRnwell. Acute muscle stretching inhibits maximal strength performance. Res. Q. Exerc. Sport. 69:411-415. 1998.

14. MuRPHY, D.R. Dynamic range of motion training: An alternative to static stretching. Chiropr. Sports Med. 8:59-66. 1994.

15. Nelson, A.G., I.K. Guillory, C. Cornwell, AND J. KoKkoNEN. Inhibition of maximal voluntary isokinetic torque production following stretching is velocity-specific. J. Strength Cond. Res. 15:241-246. 2001. 
16. NeVILL, A.M., AND G. AtKInson. Assessing agreement between measurements recorded on a ratio scale in sports medicine and sports science. Br. J. Sports Med. 31:314-318. 1997.

17. Pope, R.P., R.D. Herbert, J.D. Kirwan, and B.J. Graham. A randomized trial of preexercise stretching for prevention of lower-limb injury. Med. Sci. Sports Exerc. 32:271-277. 2000.

18. Rosenbaum, D., AND E.M. HENNIG. The influence of stretching and warm-up exercises on Achilles tendon reflex activity. $J$. Sports Sci. 13:481-90. 1995.

19. SHELlOCK, F.G., AND W.E. PRENTICE. Warming-up and stretching for improved physical performance and prevention of sports-related injuries. Sports Med. 2:267-278. 1985.

20. Unick, J., H.S. Kieffer, W. Cheesman, And A. Feeney. The acute effects of static and ballistic stretching on vertical jump performance in trained women. J. Strength Cond. Res. 19:206212. 2005.
21. WALShE, A.D., AND G.J. WiLsON. The influence of musculotendinous stiffness on drop jump performance. Can. J. Appl. Physiol. 22:117-132. 1997.

22. Witrouw, E., L. Danneels, P. Asselman, T. D'Have, and D. CAMBIER. Muscle flexibility as a risk factor for developing muscle injuries in male professional soccer players. A prospective study. Am. J. Sports Med. 31:41-46. 2003.

23. YounG, W., AND S. ElLIOTt. Acute effects of static stretching, proprioceptive neuromuscular facilitation stretching, and maximum voluntary contractions on explosive force production and jumping performance. Res. Q. Exerc. Sport. 72:273-279. 2001.

Address correspondence to Alun G. Williams, a.g. williams@mmu.ac.uk. 